\title{
Root lodging is a physical stress that changes gene expression from sucrose accumulation to degradation in sorghum
}

(1) CrossMark

\author{
Hiroshi Mizuno $^{1^{*}} \mathbb{D}$, Shigemitsu Kasuga ${ }^{2}$ and Hiroyuki Kawahigashi ${ }^{1}$
}

\begin{abstract}
Background: Sorghum (Sorghum bicolor L.) is used as a raw material for biofuels because it accumulates sugars at high levels in the stem. Lodging of sorghum occurs when the soil is wet and very high winds blow across the field. In root lodging, the roots are pulled loose from the soil, causing the plant to fall over. Lodging reduces the yield of nonstructural carbohydrates. It is not yet clear which genes show changes in expression when sorghum falls over. We compared whole-gene expression in the mature stems of intact and lodged sorghum plants, with a focus on comparisons from the perspective of differences in sugar accumulation or degradation.

Results: Lodging decreased sucrose content, starch content, and ratio of sucrose to total sugars in the stems of the sorghum cultivar SIL-05. Particular paralogs of SWEET and TMT family genes, which encode sucrose or hexose transporters, or both, were significantly highly expressed in intact or lodged sorghum stems. In intact stems, genes encoding the glucose-6-phosphate translocator, aquaporins, and enzymes involved in photosynthesis and starch synthesis were highly expressed. In lodged sorghum stems, expression of genes associated with sucrose or starch degradation or energy production was increased. Notably, expression of genes encoding enzymes catalyzing irreversible reactions and associated with the first steps of these metabolic pathways (e.g. INV, SUS, and hexokinase- and fructokinase-encoding genes) was significantly increased by lodging. Expression of SUT, SPS, and SPP was almost the same in intact and lodged sorghum.

Conclusions: Specific paralogs of sucrose-associated genes involved in metabolic pathways and in membrane transport were expressed in the stems of sorghum SIL-05 at the full-ripe stage. Root lodging drastically changed the expression of these genes from sucrose accumulation to degradation. The changes in gene expression resulted in decreases in sugar content and in the proportion of sucrose to hexoses in the stems of lodged plants.
\end{abstract}

Keywords: Biofuel, RNA-seq, Stem, Sugar content, Sugar metabolism, Sugar transporter

\section{Background}

Biofuel productivity is affected by the type of biomass used and the efficiency of its conversion from plantderived raw material to sugar [1]. The simplest and most cost-effective way to produce biofuels is to use materials containing large amounts of plant-derived sugars. Some plants, such as sorghum and sugarcane, uniquely accumulate sugar in their stems. The sorghum internode contains spacious apoplasts and vacuoles in which photosynthesized sugars are accumulated at high concentrations [2]. Sorghum therefore has high potential to become a

\footnotetext{
*Correspondence: hmizuno@affrc.go.jp

${ }^{1}$ Institute of Crop Science (NICS), National Agriculture and Food Research

Organization, 2-1-2, Kannondai, Tsukuba, Ibaraki 305-8518, Japan

Full list of author information is available at the end of the article
}

feedstock for large-scale bioenergy production. However, its sugar content and composition vary depending on the cultivar [3-5]. Sorghums with a relatively high sugar content-so called sweet sorghums-have been bred for edible and industrial use [6].

In plants, an organ-such as the leaf-that supplies photosynthetic sucrose is called a source, and an organ-such as the seed and stem-that accepts them is called a sink. In a source organ, during sucrose biosynthesis, sucrose phosphate synthase (SPS) synthesizes sucrose-6-phosphate from UDP-glucose and fructose 6-phosphate; sucrose-6-phosphate is then dephosphorylated by sucrose phosphate phosphatase (SPP) to produce sucrose. In a sink organ, translocated sucrose is stored, or degraded and used for energy metabolism, 
organ formation, synthesis of stored carbohydrates such as monosaccharides and starch, or re-synthesis of sucrose. During sucrose metabolism, sucrose synthase (SUS) degrades sucrose to UDP-glucose and fructose, or invertase (INV) degrades sucrose to glucose and fructose. UDPglucose is further converted to glucose-1-phosphate (G1P) by glucose-1-phosphate uridyltransferase; glucose is converted to glucose-6-phosphate (G6P) by hexokinase (HEX), and fructose is converted to fructose-6-phosphate by fructokinase (FRK). Nucleotide or phosphorylated sugars are activated forms of monosaccharides, which are precursors of various molecules such as starch. The starch biosynthesis pathway involves a step of ADP- $\alpha-\mathrm{D}$-glucose production by the action of glucose-1-phosphate adenylyltransferase and a repeating step of glycosyl transfer by starch synthase and further modification by the action of 1,4- $\alpha$-glucan branching enzyme.

Plasma- or vacuolar-membrane-located sugar transporters also contribute to the distribution of sucrose. Sucrose is unloaded from the phloem; once it flows out into the apoplasts it is taken up by parenchymal cells and then accumulates in vacuoles [2, 7]. It is considered that sugars will eventually be exported transporter (SWEET) and sucrose transporter (SUT) transport sucrose, and tonoplast monosaccharide transporter (TMT) transports monosaccharides, but their substrate specificity has not been completely elucidated [8-14]. Aquaporins are needed to transport water into the cell or vacuole to counteract the effect of increasing osmotic pressure with increasing sugar content. Thus, high-level sugar accumulation is achieved by the combined function of various families of enzymes and transporters.

Sugars also act as signaling molecules that control metabolism and energy production in relation to the roles of sources and sinks. Such sugars include sucrose and fructose, and glucose and its derivatives G6P, G1P, and trehalose-6-phosphate (T6P) [15]. These signaling molecules regulate sugar utilization and starch metabolism and interact with other signaling pathways, including those mediated by plant hormones [15-17]. Direct sugar sensing and signaling occur via specific sugar-binding sensors. Candidates for sugar-binding sensors include SUT for sucrose; HEX and RGS (regulator of G-protein signaling) for glucose and its derivatives; fructose-1,6bisphosphatase, FRK, and FLN (fructokinase-like protein) for fructose; and KIN10/11 (Arabidopsis protein kinase 10/11) and SnRK1 (sucrose non-fermenting related protein kinase 1) for T6P [15]. HEX, fructose1,6-bisphosphatase, FRK, and SUT are dual-function molecules that act as both sugar sensors and enzymes involved in sugar metabolism or sugar transport [18].

In sorghum, comparisons of gene expression have been performed to identify systematically the critical genes that determine stem sugar content. For example, comparisons among diverse genotypes with different sugar contents (SSV74, SPV1616, R159, Atlas, Fremont, PI152611, AR2400, and PI455230) [19, 20] have revealed that changes in the expression levels of SUT, SPS, and INV are associated with variations in the sugar contents of different sorghum cultivars. Comparison of developmental stages has indicated that the expression levels of $I N V$ and SUS decrease after anthesis, when sucrose accumulation starts in the stem [21]. Although these comparisons have helped to elucidate the diversity of genes associated with sucrose metabolism, we still need to elucidate the critical genes regulating sugar accumulation and release.

However, a number of features of sorghum make it difficult to find genes associated with sugar content. Synthesis and degradation of sucrose occur at different rates in different types of cells simultaneously, and the organ structure of stems changes developmentally during long periods of sucrose accumulation [22, 23]. Sugar content is affected by environmental conditions [24]. Moreover, a number of phenotypic traits affect sugar content, namely plant height, total dry matter, grain yield, stem diameter, number of tillers per plant, juice weight, and flowering time $[25,26]$. To identify the critical genes associated with sugar content, it is thus important, as far as is possible, to compare gene expression at the same developmental stage of the same tissue in the same cultivation environment. Therefore, we need to have an experimental method by which we can change and analyze gene expression from sucrose accumulation to degradation.

We hypothesized that root lodging (hereafter, lodging) is a physical stress that changes gene expression from sugar accumulation to degradation. Lodging of sorghum occurs when the soil is wet and very high winds blow across the field. The roots are pulled loose from the soil, causing the plant to fall over, although the stem remains intact. When lodging occurs in the early stages of the crop, the sorghum will usually re-stand, but the lodging reduces the yield of nonstructural carbohydrates [27]. It is not yet clear which genes show changes in expression when sorghum falls over. Here, we compared gene expression between intact and lodged sorghum of the cultivar SIL-05. SIL-05 accumulates sugars to relatively high levels in the stem [5] and is a promising candidate material for biofuel production. We used RNA-seq data to model the expression of specific genes supporting sugar accumulation and release in the stem. We discuss the characteristics of gene expression affected by lodging and the physiological factors in lodging stress that affect sugar metabolism.

\section{Results}

Lodging reduces Brix, sucrose percentage, and starch content

We grew 48 plants of the sorghum cultivar SIL-05 in a field. Twelve of the plants lodged at the ear-heading 
stage and were still lodged at the time of full seed ripening. Thirty-six plants remained upright. The Brix of the stem ranged from $11.1 \%$ up to $19.7 \%$ across all plants, but the lodged plants had relatively low Brix values (Fig. 1a). The sucrose ratio (the sucrose content as a percentage of the sum of sucrose, glucose, and fructose weights) ranged from $0 \%$ up to $84.9 \%$ (Fig. 1a). The sucrose content of the intact plants was greater than that of the lodged plants, whereas the glucose and fructose contents were lower (Fig. 1a). We then selected three independent typical intact (\#13, \#15, \#16) and lodged (\# 33, \# 37, \# 48) plants for further analysis (Fig. 1b). The average Brix of the intact plants was $18.7 \%$, whereas that of the lodged plants was $11.8 \%$ (Fig. 1b), indicating that sucrose had accumulated to high levels in the stems of the intact plants. The sucrose ratio in the intact plants was $71.3 \%$, whereas that in the lodged plants was $3.7 \%$ (Fig. 1b). Both the glucose content and the fructose content in the lodged plants were almost double those in the intact plants; thus, the Brix values of the lodged plants were explained nearly entirely by the plants' hexose contents. Starch content tended to be higher in intact plants than in lodged ones (Fig. 1c). There was slight but significant difference in raw panicle weight (intact > lodged), but there were no significant differences in plant height, stem diameter, or raw stem weight between lodged and intact plants (Additional file 1: Figure S1).

Identification of differentially expressed genes by RNA-seq RNA extracted from intact and lodged plants was sequenced by using a next-generation sequencer. Fragments per kilobase of exon per million mapped sequence reads (FPKM) for each gene were compared between intact and lodged plants (Fig. 2). Of a total of 27,608 genes, 1771 were significantly highly expressed in intact plants and 1866 were significantly highly expressed in lodged plants (Additional file 2: Table S1).

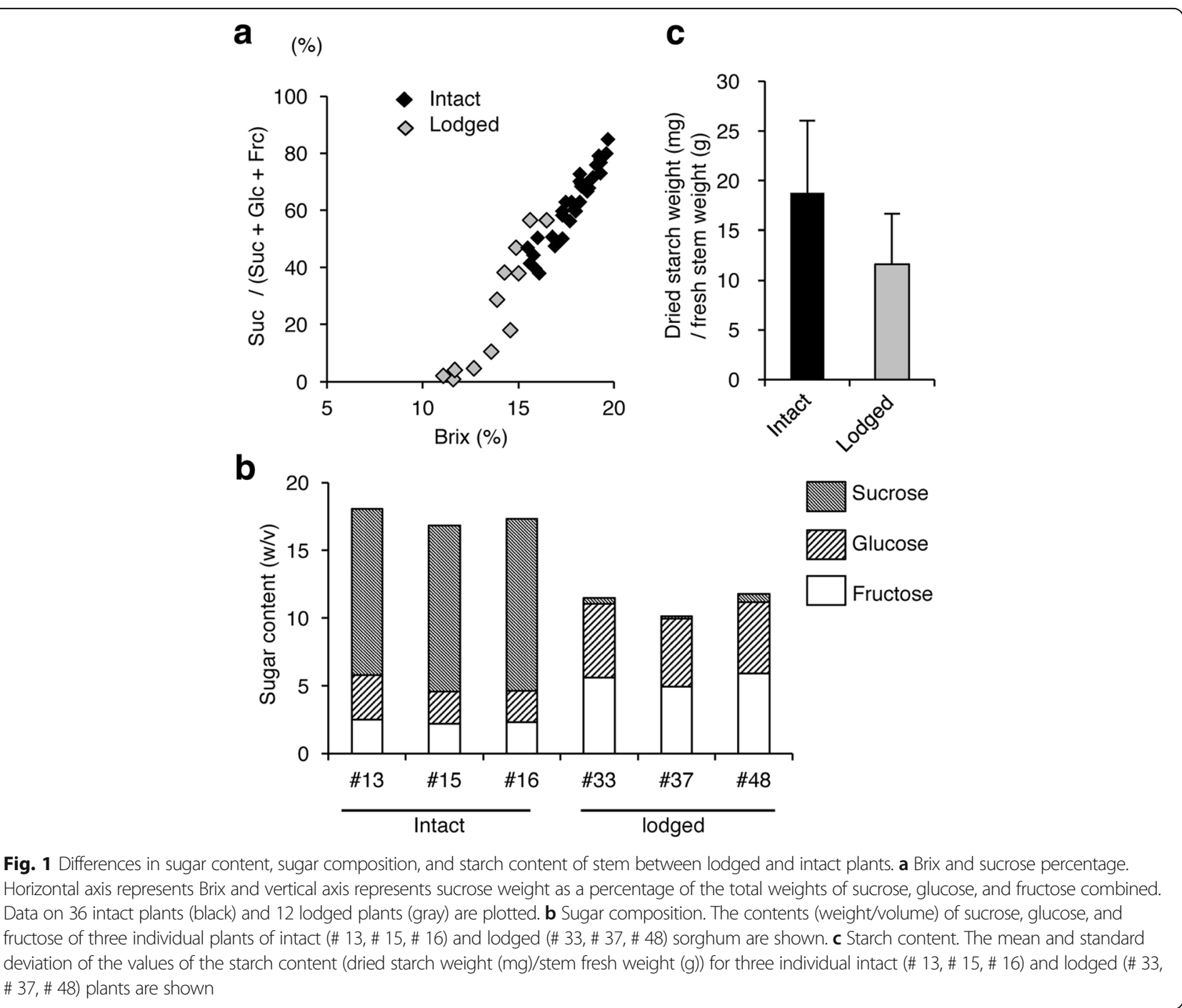




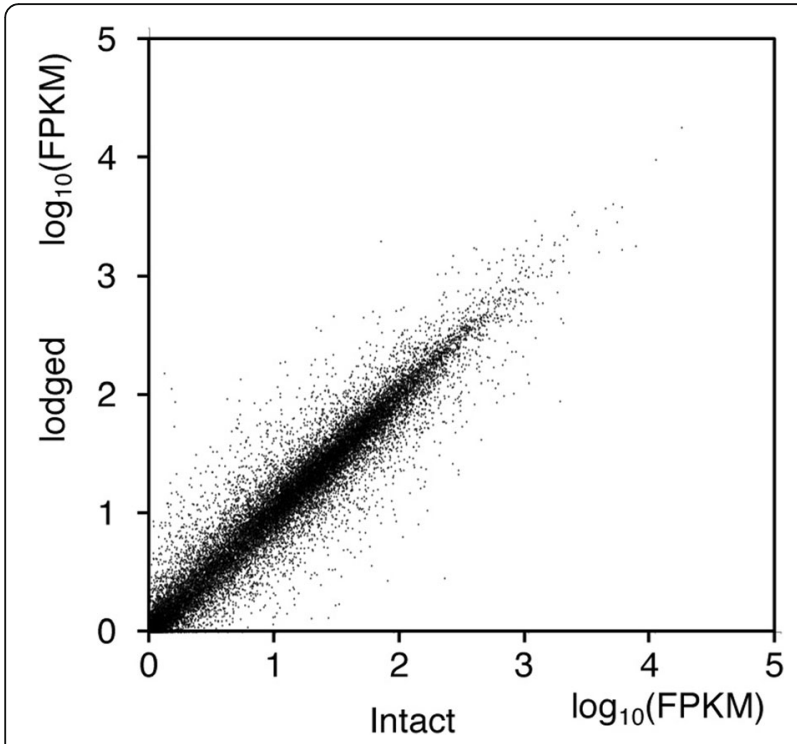

Fig. 2 Changes in gene expression level with lodging. Fragments per kilobase of exon per million mapped sequence reads (FPKM) of 27,608 genes were plotted for lodged plants (vertical axis) and intact plants (horizontal axis). Values are $\log _{10}$ of average FPKM calculated from three individual plants: intact (\# 13, \# 15, \# 16) or lodged (\# 33, \# 37, \# 48)

\section{Overview of metabolic pathways activated in intact and} lodged sorghum plants

The relative FPKM of lodged and intact plants were mapped on the metabolic pathways. In intact plants, expression of genes involved in sucrose or starch synthesis or the Calvin cycle was high (Fig. 3: pathways 1 to 3). In lodged plants, expression of genes involved in the pentose phosphate pathway, the TCA cycle, the fermentation pathway, and the trehalose pathway (Fig. 3: pathways 4 to 7 ) was high. Different paralogs involved in sucrose degradation or starch degradation were expressed at high levels in intact or lodged plants (Fig. 3, pathways 8 and 9).

\section{Expression of genes encoding enzymes involved in sucrose and starch metabolic pathways \\ Sucrose synthesis}

Sucrose biosynthesis is a two-step enzymatic reaction starting from UDP-glucose. Expression of the genes involved in sucrose biosynthesis was barely changed by lodging. Of five SPS and three SPP paralogs, only the expression of one SPS (Sb05g007310.1) was significantly higher in intact than in lodged sorghum stem (Fig. 4a).

\section{Sucrose degradation}

Sucrose degradation is catalyzed by either INV (EC 3.2.1.26) or SUS (EC 2.4.1.13). In lodged plants, expression of INV (Sb04g021810.1 and Sb06g031930.1) and SUS (Sb01g033060.1 and Sb10g006330.1) was significantly higher than in intact plants (Fig. 4b). Expression of the genes encoding hexokinase (Sb09g005840.1 and Sb09g026080.1) and fructokinase (Sb03g030470.1 and Sb07g001320.1) was also significantly higher in lodged stems (Fig. 4b).

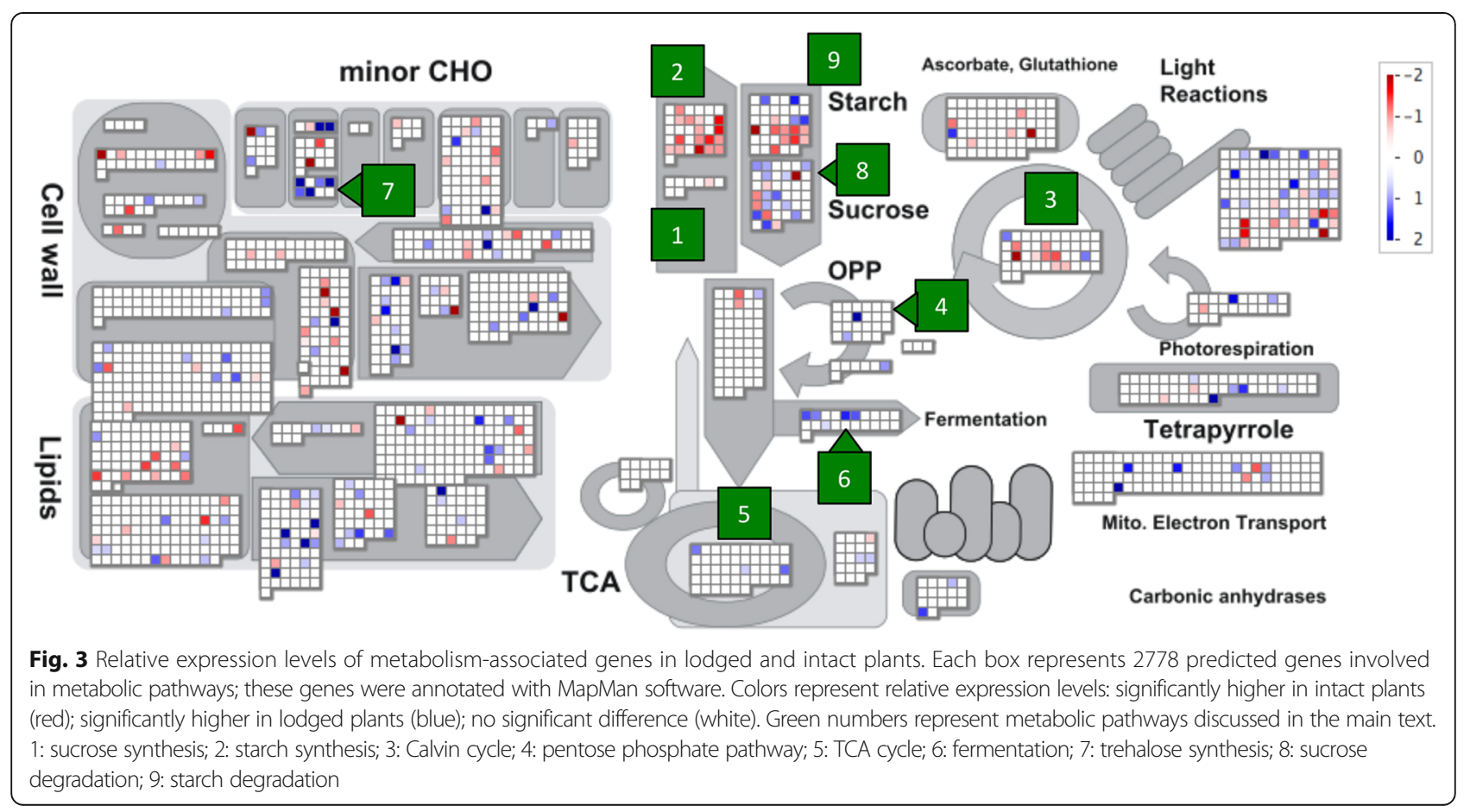




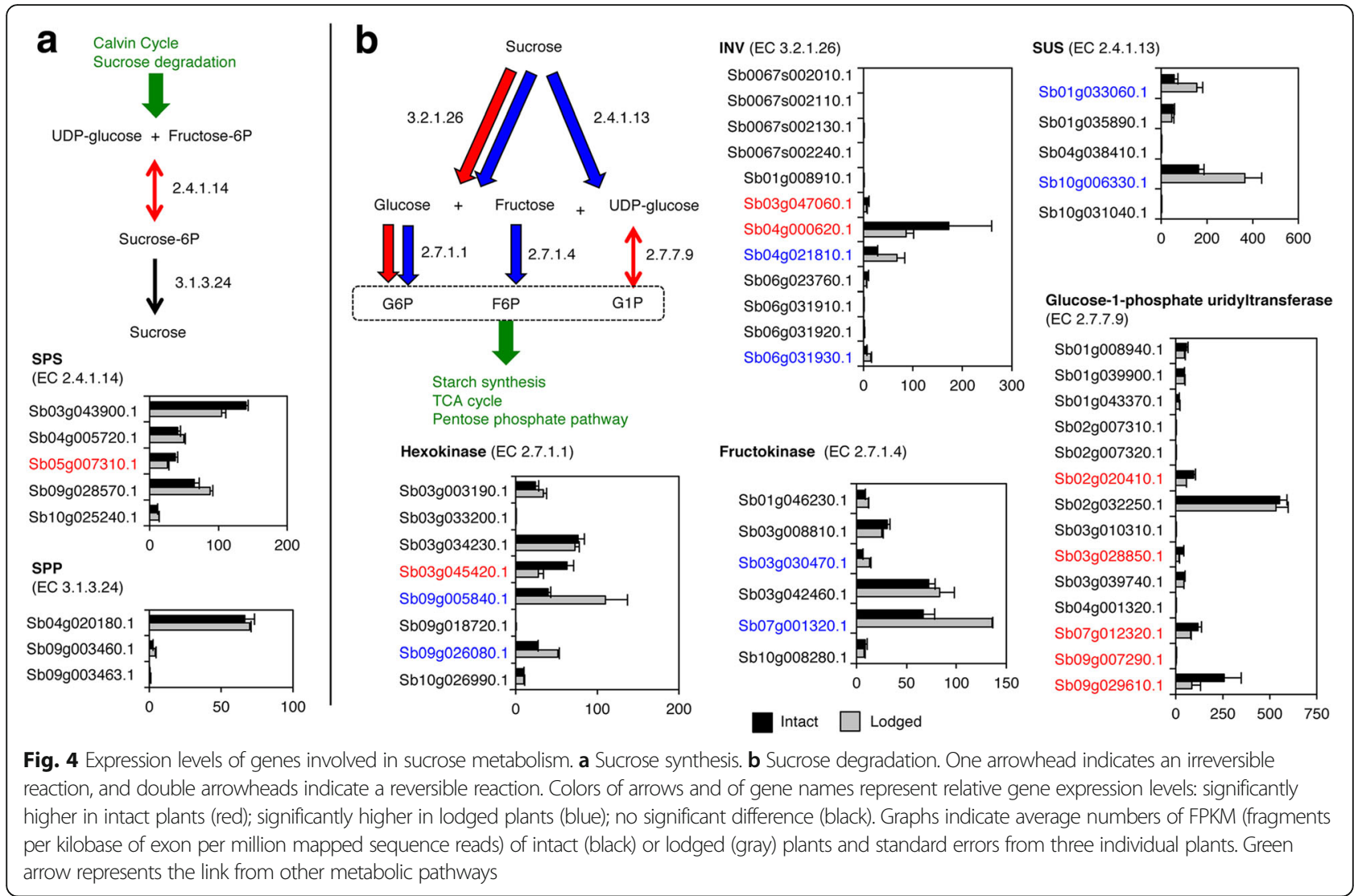

\section{Starch synthesis}

Genes encoding enzymes involved in starch biosynthesis steps were expressed at significantly higher levels in the stems of intact sorghum than in those of lodged plants (Fig. 5a). Notably, genes encoding fructose-1,6-biphosphatase (EC 3.1.3.11), glucose-1-phosphate adenylyltransferase (EC 2.7.7.27), starch synthase (EC 2.4.1.21) and 1,4-alpha glucan branching enzyme (EC 2.4.1.18) were expressed at higher levels in intact plants than in lodged plants.

\section{Starch degradation}

There are 16 putative genes encoding $\alpha$-amylases and 16 $\beta$-amylases in the sorghum genome. Different paralogs putatively encoding $\alpha$-amylases or $\beta$-amylases were expressed at high levels in intact or lodged sorghum (Fig. 5b).

\section{Photosynthesis}

In lodged plants, expression of genes involved in the synthesis of sucrose via photosynthesis was decreased. In the case of the Calvin cycle, expression of genes for phosphoribulokinase (EC 2.7.1.19; Sb04g030950.1), fructose-1,6bisphosphatase (EC 3.1.3.11; Sb03g040900.1), and fruc tose-bisphosphate aldolase (EC 4.1.2.13; Sb05g004590.1) was highly $(\mathrm{FPKM}>100)$ and significantly decreased by lodging (Additional file 3: Figure S2). Expression of genes associated with $\mathrm{C} 4$ photosynthesis (encoding phosphoenolpyruvate carboxylase (EC 4.1.1.31), malate dehydrogenase (EC 1.1.1.82), and pyruvate-phosphate dikinase (EC 2.7.9.1)) was affected little by lodging (Additional file 4: Figure S3).

\section{Construction of models of expression of critical genes associated with sugar storage and release in stems (intact or lodged) \\ Stem (intact)}

Sucrose is translocated from the leaves into the sink organ, namely the stem. Sucrose is accumulated in the vacuoles or is degraded to hexoses in the cytosol. These hexoses are stored or used for starch synthesis. Among the genes for sucrose transporters, the expression of SWEET genes (Sb01g035840.1, Sb03g032190.1, Sb0 4g012910.1, Sb05g018110.1) was significantly higher in intact stems than in lodged ones (Fig. 6). Both SWEET4-3 (Sb04g015420.1.1) and SbSUT1 (Sb01g 045720.1.1) were highly expressed in the stem at the sucrose-accumulation stage [28], but their expression was not changed by lodging (Fig. 6). Among the genes for hexose transporters, TMTs (Sb01g030430.1, Sb01g 044010.1, Sb04g008150.1) were expressed highly in the stem; Sb04g008150.1 was expressed at significantly 


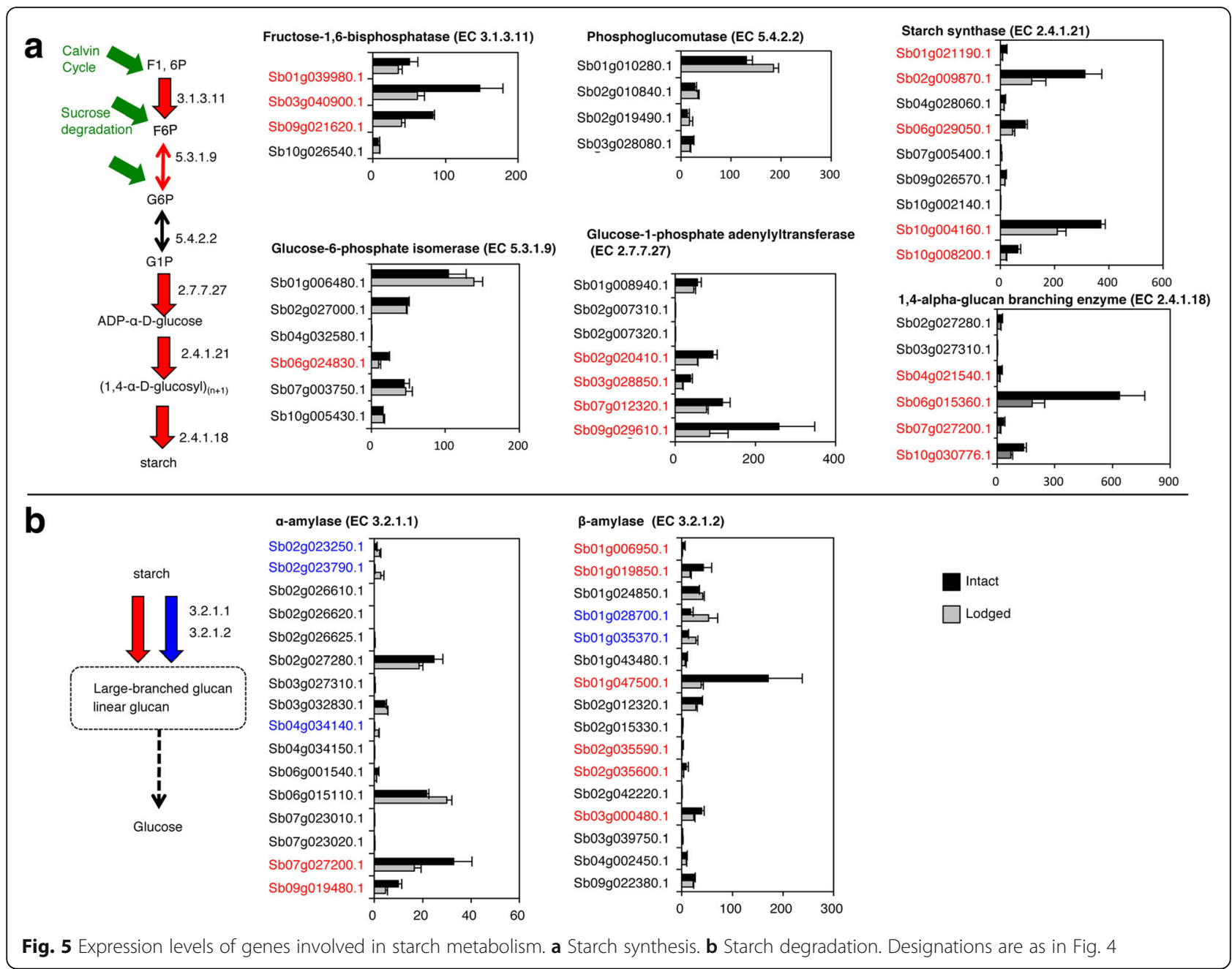

higher levels in intact plants than in lodged ones (Fig. 6).

For water transport, 10 putative aquaporin genes were expressed at significantly higher levels in intact than in lodged plants, and three of them (Sb04g028020.1, Sb06g025150.1 and Sb10g008090.1) were highly expressed (Fig. 6). Three aquaporin genes (Sb01g047140.1, Sb02g010760.1, and Sb04g032430.1) were highly expressed at the same levels in both intact and lodged stems (Fig. 6).

Expression of SPS and SPP genes was barely changed by lodging (Fig. 4). In the case of starch synthesis, 4 of 7 genes encoding glucose-1-phosphate adenylyltransferases, 5 of 9 genes encoding starch synthases, and 4 of 6 genes encoding 1,4- $\alpha$-glucan branching enzymes were expressed at significantly higher levels in the intact stem than in the lodged stem (Fig. 5a). One glucose 6-phosphate/phosphate translocator2 (GPT2) (Sb02g034980.1) was expressed at much higher levels in intact than in lodged stems (Fig. 6). These findings were summarized as a model in Fig. 7a.

\section{Stem (lodged)}

Sucrose stored or synthesized is translocated to other tissues. For sucrose transport, the expression of SWEETs (Sb03g041740.1, Sb08g013840.1, Sb08g014040.1) was increased by lodging; the expression level of SbSWEET3-8 (Sb03g041740.1) was especially high (Fig. 6). For degradation of stored sucrose, expression of INV (Sb04 g021810.1, Sb06g031930.1) was significantly higher in lodged stems than in intact ones (Fig. 4b). For hexose transport, expression of TMTs (Sb02g037570.1, Sb02g037580.1, Sb04g038440.1) was significantly increased by lodging (Fig. 6). For stored starch degradation, expression of some putative genes encoding $\alpha$-amylases and $\beta$-amylases was high (Fig. $5 b)$. For hexose phosphorylation, expression of some genes encoding hexokinases and fructokinases was increased significantly in lodged plants (Fig. 4b). We consider that these phosphorylated sugars eventually become sucrose and are translocated to other organs for use in energy-releasing glycolysis. The levels of expression of these genes were consistent with our biochemical quantification that lodged 


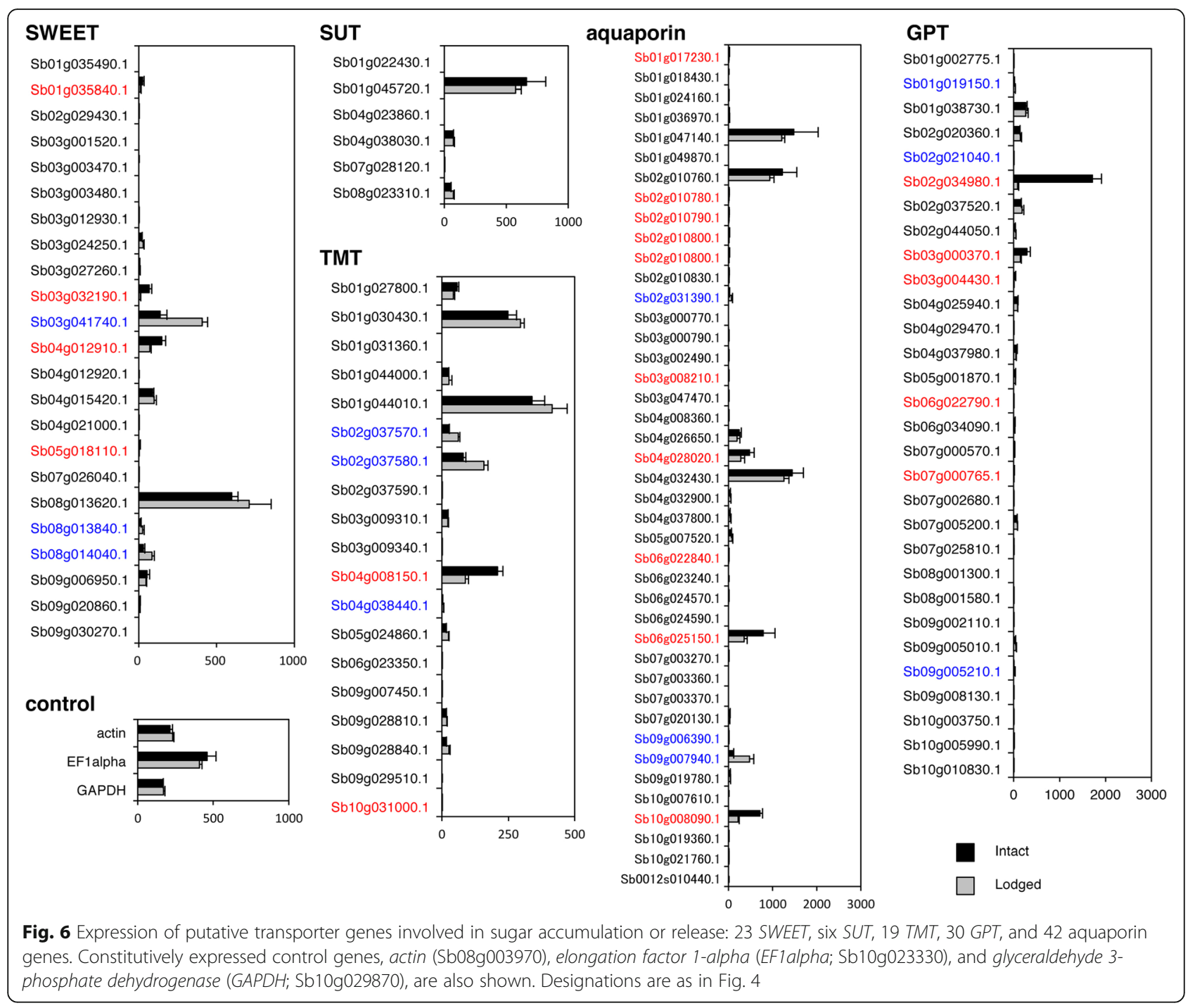

stems had lower sugar and starch contents and lower sucrose percentages than intact stems (Fig. 1a, b). These findings were summarized as a model in Fig. $7 \mathrm{~b}$.

\section{Discussion}

\section{Characteristics of sugar-associated genes affected by} lodging

To our knowledge, our study is the first to compare gene expression in the stems of intact sorghum plants with that in the stems of lodged sorghum plants. We focused on comparisons from the perspective of differences in sugar accumulation or degradation, instead of comparisons of different organs, developmental stages, or genetic variations with different sugar contents $[19,20,29,30]$. Lodging was a physical stress greatly affecting the expression of sugar-associated genes at the same developmental stage, in the same tissue, in field environments that were as similar as possible. We revealed that lodging changed sugar content and composition in the stems of the sorghum SIL-05 (Fig. 1). This was consistent with the results of our transcriptome analysis indicating that gene expression was changed from sucrose synthesis to degradation by lodging; in other words, cell status changed from energy storage to consumption by lodging (Figs. 2, 3, 4, 5, 6 and 7). We thus concluded that lodging was a physical stress that changes gene expression from sugar accumulation to degradation.

Changes in stem-specific gene expression reflect the transport of molecules to sugar storage compartments such as the apoplast and vacuoles in stem. SWEET or TMT paralogs differentially expressed upon lodging (Fig. 6) might transport sugars to and from stem-specific compartments (uptake or efflux) in accordance with changes in the demand for, and supply of, cell energy (Fig. 7). In contrast, the expression levels of SUT, SPS, and SPP were almost the same in intact and lodged plants (Figs. 4a and 6). This suggests that expression of SUT, SPS, and SPP is necessary for sucrose accumulation but does not change during the 


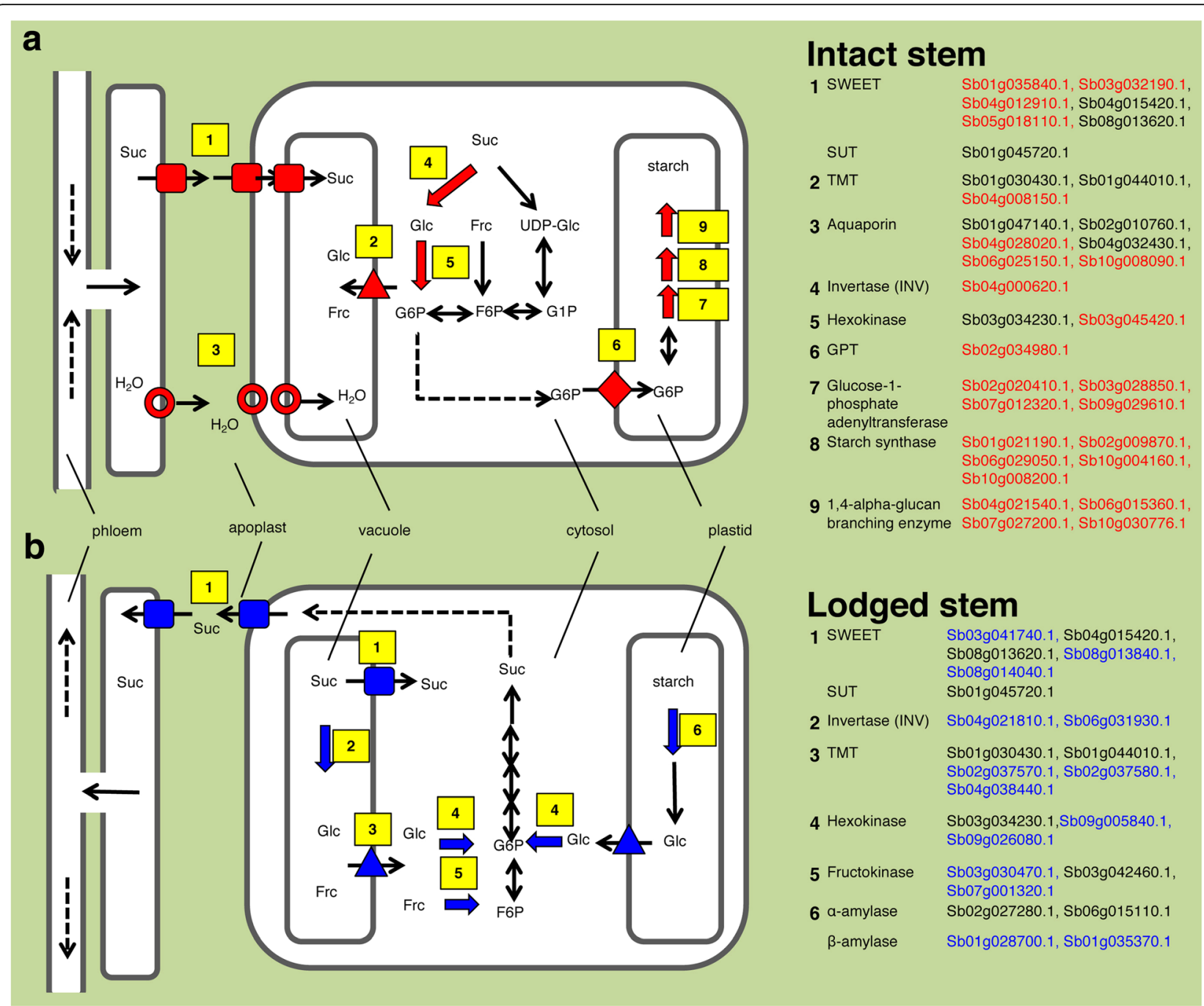

Fig. 7 Representative genes involved in sugar accumulation or release. a Model of intact sorghum. Sucrose (Suc) is accumulated in the vacuoles or is degraded to glucose (Glc) and fructose (Frc) in the cytosol. These hexoses are stored or used for starch synthesis. Genes for sucrose accumulation were highly expressed in intact stems. b Model of lodged sorghum. Sucrose stored in vacuoles is translocated to other tissues. Sucrose is synthesized from stored starch or hexoses. Genes for release of stored sucrose to other tissues were expressed in the lodged stem. Illustrations on the left show schematically the enzymatic reactions or transport steps involved in sugar accumulation or release. Yellow numbers correspond to the numbers in the tables at right. In these tables, genes within each family with expression levels that changed with lodging (red: significantly higher in intact plants; blue: significantly higher in lodged plants) and genes that were expressed at high levels but not differentially between intact and lodged plants (black) are listed

regulation of sugar metabolism. The expression level of SUT among different cultivars with different sugar content is controversial: it differs between Rio and BTx623 [31], but it does not differ between Wray and Macia [32]. We therefore consider that expression of the differentially expressed SWEET or TMT genes is regulated respectively to accumulate or release sugar in the stem of SIL-05.

Whether stored molecules are kept or degraded is often determined by the action of the first enzymes in the metabolic pathway, as usually this step is irreversible. INV enzymes are the first to degrade sucrose. At the early stage of sucrose accumulation ( 1 to 36 days after heading), INVs were barely expressed in the stem [28]. $I N V$ expression was upregulated by lodging (Fig. 4b). These data suggest that INV activity does not occur in the stem at the sucrose accumulation stage, but that INV activity is required to generate energy in the case of lodging. This is strongly supported by the finding that $I N V$ expression rapidly decreases after anthesis in the sorghum Della [21].

To convert stored hexoses into various molecules, they must first be converted to activated intermediates. Both hexokinase and fructokinase phosphorylate hexoses to produce high-energy intermediates, and those genes 
were highly expressed in lodged sorghum (Fig. 4b). We therefore consider that lodging-induced expression of these genes encoding hexokinases and fructokinases helps supply stored sugars to sugar metabolic pathways such as glycolysis.

As many enzymes involved in sugar synthesis and degradation are the same, the direction of the pathway is determined by a limited number of irreversible enzymatic reactions. In the pathway from hexoses to pyruvate for the TCA cycle and its reverse pathway, there are two irreversible steps. A total of four enzymes are responsible for these irreversible reactions, namely 6-phosphofructokinase (EC 2.7.1.11), fructose-1,6-bisphosphatase (EC 3.1.3.11), phosphoenolpyruvate synthase (EC 2.7.9.2), and pyruvate kinase (EC 2.7.1.40). Of the genes encoding these four, only the genes encoding fructose-1,6-bisphosphatase were expressed at significantly higher levels in intact than in lodged sorghum stems (Fig. 5a; Additional file 5: Figure S4). We therefore consider that the level of production of fructose-1,6-bisphosphatase helps to determine the direction of sucrose synthesis or glycolysis in photosynthetic cells.

Our molecular-based knowledge of sink organs has been obtained mainly by using the seeds or tubers of model plants such as Arabidopsis, rice, maize, and potato [1, 10, 12-14]. However, as these model plants do not accumulate sugar at high levels in the stem, we consider that the sugar-associated genes of these model plants do not functionally correspond to orthologs in sorghum that are critical for stem sugar accumulation. The sugar-transport mechanism of the sugarcane internode is symplastic unloading [33], which differs from the apoplastic unloading in sorghum [2]. In addition, the sugarcane genome is very complex owing to its high polyploidy and aneuploidy, with 10 to 12 copies of homeologous genes [34]; this is another reason why sugarcane genes do not necessarily correspond to sorghum orthologs critical for stem sugar accumulation. Sucrose-metabolism-associated gene families (SUS, SPS, SPP, and glucose-1-phosphate uridylyltransferase) have diversified because of differences in evolutionary history, as their host genomes have experienced differential rates of whole-genome duplication, tandem and segmental duplication, or mobile-elementmediated gene gain and loss [35]. Transporter genes are more diverse than sugar metabolism genes, and the evolutionary divergence of sugar transporter genes is associated with differences in the degree of sugar accumulation in the storage tissues of grasses and eudicots [36]. Moreover, SbSWEET4-3, which is constitutively highly expressed in the stem, was generated after the divergence of rice and sorghum [28]. Thus, the sugar-associated genes found in this study might have evolved as specific to sorghum.

\section{Physiological factors in lodging stress that affect sugar metabolism}

Access to light may be hindered in lodged plants. In the lodged plants, expression of genes associated with the Calvin cycle decreased (Additional file 3: Figure S2). This supports a decline in photosynthetic capacity of the lodged plants. We consider that, unlike the intact plants, the lodged plants were in a state of energy shortage; accumulated sugars and starch were therefore degraded to compensate for this shortage.

Because, in the lodged plants, oxygen consumed through respiration was not adequately supplied, the intracellular oxygen concentration of the tissues of the lodged plants may have decreased locally, resulting in hypoxia. The expression of genes putatively involved in fermentation (alcohol dehydrogenase $(A D H)$, pyruvate decarboxylase and L-lactate dehydrogenase) was increased by lodging (Fig. 3). The expression of $A D H$ genes in maize increases in hypoxia [37]. The putative sorghum orthologs (Sb01g008730, Sb05g009350) of the maize hypoxia-induced $A D H 1 / 2$ were relatively highly expressed in lodged sorghum (Additional file 2: Table S1). We therefore consider that fermentation was activated under intracellular anaerobic conditions in lodged plants.

A change caused in the concentration of sugar molecules by lodging may act as a signal to regulate sugar metabolism and energy production in the whole plant. In the lodged plants, the proportion of hexoses (glucose, fructose) to sucrose was increased (Fig. 1). The changes in expression levels of $I N V$, SUS, and the genes encoding hexokinase, fructokinase, and glucose-1-phosphate uridyltransferase with lodging (Fig. 4b) may thus contribute to the regulation of sugar metabolism and energy production in sources and sinks through the generation and/or the sensing of signaling molecules (glucose, fructose, G6P, and G1P). T6P may be another signaling molecule [16, 17]. We found that genes involved in trehalose metabolism were upregulated by lodging (Fig. 3): the expression of 6 of 15 putative T6P synthase (TPS)-encoding genes (Sb09g025660.1, Sb07g020270.1, Sb0 7g021920.1, Sb04g035560.1, Sb02g023610.1, Sb02g02 4140.1) was significantly increased by lodging (Additional file 2: Table S1). Although we did not examine the occurrence of T6P, the increase in TPS expression suggests that the concentration of T6P increased in lodged plants. Identification of sugar molecules that function as signals in sorghum and of the corresponding binding sensors is an important future goal.

Other studies have demonstrated how physical stress can change sugar metabolism. Wounding induces the expression of $I N V$, followed by hexose accumulation and post-harvest sucrose loss, in sugar beet [38]. Drought or salt stress can induce the expression of sugar transporter 
genes in Arabidopsis [39]. Comparison with the responses to other types of physical stress will elucidate the diversity of the physical stresses that affect the expression of genes involved in sugar metabolism.

\section{Conclusions}

Specific paralogs of sucrose-associated genes involved in metabolic pathways and in membrane transport were expressed in the stems of sorghum SIL-05 at the fullripe stage. Root lodging drastically changed the expression of these genes from sucrose accumulation to degradation. The changes in gene expression resulted in decreases in sugar content and in the proportion of sucrose to hexoses in the stems of lodged plants.

\section{Methods}

\section{Plant materials and quantification of sugar and starch contents}

Seeds of sorghum cultivar SIL-05 were sown on 26 May 2012 in a field at Shinshu University in Nagano, Japan. Weather-induced lodging occurred in 12 of the 48 plants just after ear-heading in late August 2012. The lodged plants were still lodged at the time of sampling of their internodes on 10 October 2012. Internodes located in the central part of each plant were collected. The collected internodes were completely developed and the seeds were fully ripe. For quantification of sugars, juice at the internode was obtained by using a French press. The Brix of the sorghum juice was measured with a PAL-1 refractometer (Atago, Tokyo, Japan). Sugar contents (sucrose, glucose, and fructose) were measured by capillary electrophoresis [5]. For quantification of starch, frozen internodes were powdered, shaken with $0.2 \%$ $\mathrm{NaOH}$ for $30 \mathrm{~min}$, and stored overnight at $4{ }^{\circ} \mathrm{C}$. The extract was filtered through gauze to remove the insoluble residual fibers. The filtered extract was centrifuged and the starch pellet was washed stepwise with $0.2 \% \mathrm{NaOH}$, distilled water, and methanol and then dried. The dried starch weight $(\mathrm{mg}) / \mathrm{stem}$ fresh weight $(\mathrm{g})$ ratios were compared between intact and lodged plants.

\section{RNA-seq}

Sorghum stems were immediately frozen in liquid nitrogen, fragmented, and mixed to minimize the effect of transcriptome unevenness among stem regions. RNA was extracted with an RNeasy plant kit (Qiagen, Hilden, Germany). RNA quality was calculated by using the Bioanalyzer 2100 algorithm (Agilent Technologies, Palo Alto, CA, USA); high-quality (RNA integrity number > 8) RNA was used for RNA-seq. cDNAs were synthesized from RNAs [40, 41] and 100-bp reads were obtained by using an Illumina Hiseq2000 sequencer (Illumina, San Diego, CA, USA). Low-quality nucleotides $(<\mathrm{Q} 15)$ from both the $5^{\prime}$ - and the $3^{\prime}$-ends were trimmed by using btrim [42]. Reads were aligned to the reference genome of BTx623 (Sbicolor_v1.4_79) [43] by using TopHat version 2.0.4 [44]. Differentially expressed genes were identified by using Cuffdiff version 2.2.0 [45], with the gene models annotated in Sbicolor ver1.4 [46].

\section{Mapping genes on metabolic pathways}

MapMan (3.5.1 R2) software [47] was used to map the ratio of expression (lodged to intact) of each gene to known metabolic pathways. The $\log _{2}$ value was calculated after adding 1 to the FPKM of each gene. Lists of enzyme-encoding genes belonging to each metabolic pathway were downloaded from SorghumCyc ver 1.1 [48] and manually modified.

\section{Additional files}

Additional file 1: Figure S1. Comparison of sizes and weights in intact and lodged sorghum. Average plant height, stem diameter, raw stem weight, and raw panicle weight of 36 intact plants and 12 lodged plants are shown. (PDF $27 \mathrm{~kb}$ )

Additional file 2: Table S1. Comparison of expression levels of sorghum genes in intact and lodged stems. Columns show the following, from the left: gene name (gene_id), chromosomal position (locus), average FPKM (fragments per kilobase of exon per million mapped sequence reads) of three intact sorghum plants (fpkm_intact), average FPKM of three lodged sorghum plants (fpkm_lodged), $\log _{2}$ value of fold change from lodged sorghum to intact sorghum (log2_ratio), P-value (p_value), q-value (q_value), and statistical significance (yes/no). (XLSX $2174 \mathrm{~kb})$

Additional file 3: Figure S2. Comparison of expression levels of genes involved in the Calvin cycle in intact and lodged stems. Columns show the following, from the left: gene name, reaction EC, average FPKM of three intact plants (FPKM_intact), average FPKM of three lodged sorghum plants (FPKM_lodged), and statistical significance (yes/no). (PDF $42 \mathrm{~kb}$ )

Additional file 4: Figure S3. Comparison of expression levels of genes involved in C4 photosynthesis in intact and lodged stems. Designations are as in Additional file 3: Figure S2. (PDF $37 \mathrm{~kb}$ )

Additional file 5: Figure S4. Expression of genes involved in irreversible steps of sugar metabolism. One arrowhead indicates an irreversible reaction, and double arrowheads indicate a reversible reaction. Colors of arrows and of gene names represent relative gene expression levels: significantly higher in intact plants (red); no significant difference (black). Graphs indicate average FPKM of intact (black) or lodged (gray) plants and standard errors from three individual plants. (PDF $481 \mathrm{~kb}$ )

\section{Abbreviations \\ FPKM: Fragments per kilobase of exon per million mapped sequence reads; FRK: Fructokinase; G1P: Glucose-1-phosphate; G6P: Glucose-6-phosphate; GPT: Glucose 6-phosphate/phosphate translocator; HEX: Hexokinase; INV: Invertase; SPP: Sucrose phosphate phosphatase; SPS: Sucrose phosphate synthase; SUS: Sucrose synthase; SUT: Sucrose transporter; SWEET: Sugars will eventually be exported transporters; T6P: Trehalose-6-phosphate; TMT: Tonoplast monosaccharide transporter; TPS: T6P synthase}

\section{Acknowledgments}

We thank Mr. Takayuki Yazawa of the National Institute of Agrobiological Sciences for his advice on bioinformatics.

\section{Funding}

This work was supported financially by the Institute of Crop Science, National Agriculture and Food Research Organization. The funding body did not participate in study design, collection, analysis or interpretation of data, or writing the manuscript. 


\section{Availability of data and materials}

Seeds are available from the National Agriculture and Food Research Organization in Japan on reasonable request. The RNA-seq datasets are available in the DDBJ (DNA Data Bank of Japan) Sequence Read Archive [Accession Nos. DRX013493 (\# 13), DRX013494 (\# 15), DRX013495 (\# 16), DRX013497 (\# 33), DRX013498 (\# 37), and DRX013499 (\# 48)].

\section{Authors' contributions}

HM and HK designed the experiments. SK provided the seeds, and quantified sucrose contents of the internodes. HK extracted the RNA and quantified the starch content. HM performed data analysis and drafted the manuscript. All authors have read and approved the final manuscript.

\section{Ethics approval and consent to participate}

Not applicable.

\section{Consent for publication}

Not applicable.

\section{Competing interests}

The authors declare that they have no competing interests.

\section{Publisher's Note}

Springer Nature remains neutral with regard to jurisdictional claims in published maps and institutional affiliations.

\section{Author details}

${ }^{1}$ Institute of Crop Science (NICS), National Agriculture and Food Research Organization, 2-1-2, Kannondai, Tsukuba, Ibaraki 305-8518, Japan. ${ }^{2}$ Faculty of Agriculture, Shinshu University, 8304 Minami-minowa, Nagano 399-4598, Japan

Received: 8 August 2017 Accepted: 19 December 2017

Published online: 03 January 2018

\section{References}

1. Patrick JW, Botha FC, Birch RG. Metabolic engineering of sugars and simple sugar derivatives in plants. Plant Biotechnol J. 2013;11(2):142-56.

2. Tarpley L, Vietor DM. Compartmentation of sucrose during radial transfer in mature sorghum culm. BMC Plant Biol. 2007:7:33.

3. Wang ML, Zhu CS, Barkley NA, Chen ZB, Erpelding JE, Murray SC, et al. Genetic diversity and population structure analysis of accessions in the US historic sweet sorghum collection. Theor Appl Genet. 2009;120(1):13-23.

4. Murray SC, Rooney WL, Hamblin MT, Mitchell SE, Kresovich S. Sweet sorghum genetic diversity and association mapping for brix and height Plant Genome-Us. 2009;2(1):48-62.

5. Kawahigashi H, Kasuga S, Okuizumi H, Hiradate S, Yonemaru J. Evaluation of Brix and sugar content in stem juice from sorghum varieties. Grassl Sci. 2013:59(1):11-9.

6. Calvino M, Messing J. Sweet sorghum as a model system for bioenergy crops. Curr Opin Biotech. 2012;23(3):323-9.

7. Ayre BG. Membrane-transport systems for sucrose in relation to whole-plant carbon partitioning. Mol Plant. 2011;4(3):377-94.

8. Eom JS, Chen LQ, Sosso D, Julius BT, Lin IW, XQ Q, et al. SWEETs, transporters for intracellular and intercellular sugar translocation. Curr Opin Plant Biol. 2015;25:53-62

9. Baker RF, Leach KA, Braun DM. SWEET As sugar: new sucrose effluxers in plants. Mol Plant 2012;5(4):766-768.

10. Doidy J, Grace E, Kuhn C, Simon-Plas F, Casieri L, Wipf D. Sugar transporters in plants and in their interactions with fungi. Trends Plant Sci. 2012;17(7): 413-22.

11. Lalonde S, Wipf D, Frommer WB. Transport mechanisms for organic forms of carbon and nitrogen between source and sink. Annu Rev Plant Biol. 2004:55:341-72.

12. Chen LQ, Cheung LS, Feng L, Tanner W, Frommer WB. Transport of sugars. Annu Rev Biochem. 2015;84:865-94.

13. Kuhn C, Grof CPL. Sucrose transporters of higher plants. Curr Opin Plant Biol. 2010;13(3):287-98.

14. Williams LE, Lemoine R, Sauer N. Sugar transporters in higher plants - a diversity of roles and complex regulation. Trends Plant Sci. 2000;5(7):283-90.
15. Li L, Sheen J. Dynamic and diverse sugar signaling. Curr Opin Plant Biol. 2016:33:116-25.

16. Paul M. Trehalose 6-phosphate. Curr Opin Plant Biol. 2007;10(3):303-9.

17. Gomez LD, Baud S, Gilday A, Li Y, Graham IA. Delayed embryo development in the ARABIDOPSIS TREHALOSE-6-PHOSPHATE SYNTHASE 1 mutant is associated with altered cell wall structure, decreased cell division and starch accumulation. Plant J. 2006;46(1):69-84.

18. Aguilera-Alvarado GP, Sanchez-Nieto S. Plant hexokinases are multifaceted proteins. Plant Cell Physiol. 2017;58(7):1151-60.

19. Qazi HA, Paranjpe S, Bhargava S. Stem sugar accumulation in sweet sorghum - activity and expression of sucrose metabolizing enzymes and sucrose transporters. J Plant Physiol. 2012;169(6):605-13.

20. Shakoor N, Nair R, Crasta O, Morris G, Feltus A, Kresovich SA. Sorghum Bicolor expression atlas reveals dynamic genotype-specific expression profiles for vegetative tissues of grain, sweet and bioenergy sorghums. BMC Plant Biol. 2014;14

21. McKinley B, Rooney W, Wilkerson C, Mullet J. Dynamics of biomass partitioning stem gene expression, cell wall biosynthesis, and sucrose accumulation during development of Sorghum Bicolor. Plant J. 2016:88(4):662-80.

22. Milne RJ, Offler CE, Patrick JW, Grof CPL. Cellular pathways of source leaf phloem loading and phloem unloading in developing stems of Sorghum Bicolor in relation to stem sucrose storage. Funct Plant Biol. 2015;42(10):957-70.

23. Milne RJ, Perroux JM, Rae AL, Reinders A, Ward JM, Offler CE, et al. Sucrose transporter localization and function in phloem unloading in developing stems. Plant Physiol. 2017;173(2):1330-41.

24. Ogbaga CC, Stepien P, Dyson BC, Rattray NJW, Ellis DI, Goodacre R, et al. Biochemical analyses of sorghum varieties reveal differential responses to drought. PLoS One. 2016;11(5)

25. Shiringani AL, Frisch M, Friedt W. Genetic mapping of QTLs for sugar-related traits in a RIL population of Sorghum Bicolor L. Moench. Theor Appl Genet. 2010;121(2):323-36.

26. Ritter KB, Jordan DR, Chapman SC, Godwin ID, Mace ES, McIntyre CL. Identification of QTL for sugar-related traits in a sweet x grain sorghum (Sorghum Bicolor L. Moench) recombinant inbred population. Mol Breeding .2008:22(3):367-84

27. Fedenko JR, Erickson JE, Singh MP. Root lodging affects biomass yield and carbohydrate composition in sweet sorghum. Ind Crop Prod. 2015;74:933-8.

28. Mizuno H, Kasuga S, Kawahigashi $\mathrm{H}$. The sorghum SWEET gene family: stem sucrose accumulation as revealed through transcriptome profiling. Biotechnol Biofuels. 2016;9:127.

29. Chandra A, Verma PK, Islam MN, Grisham MP, Jain R, Sharma A, et al. Expression analysis of genes associated with sucrose accumulation in sugarcane (Saccharum spp. hybrids) varieties differing in content and time of peak sucrose storage. Plant Biol. 2015;17(3):608-17.

30. Papini-Terzi FS, Rocha FR, Vencio RZN, Felix JM, Branco DS, Waclawovsky AJ, et al Sugarcane genes associated with sucrose content. BMC Genomics. 2009;10:120.

31. Milne RJ, Byrt CS, Patrick JW, Grof CPL. Are sucrose transporter expression profiles linked with patterns of biomass partitioning in sorghum phenotypes? Front Plant Sci. 2013;4:223.

32. Bihmidine S, Baker RF, Hoffner C, Braun DM. Sucrose accumulation in sweet sorghum stems occurs by apoplasmic phloem unloading and does not involve differential sucrose transporter expression. BMC Plant Biol. 2015;15:186.

33. Rae AL, Perroux JM, Grof CPL. Sucrose partitioning between vascular bundles and storage parenchyma in the sugarcane stem: a potential role for the ShSUT1 sucrose transporter. Planta. 2005;220(6):817-25.

34. Souza GM, Berges $H$, Bocs S, Casu R, D'Hont A, Ferreira JE, et al. The Sugarcane Genome Challenge: Strategies for Sequencing a Highly Complex Genome. Tropical Plant Biol. 2011:4(3-4):145-56.

35. Jiang SY, Chi YH, Wang JZ, Zhou JX, Cheng YS, Zhang BL, et al. Sucrose metabolism gene families and their biological functions. Sci Rep-Uk. 2015;5:17583.

36. Wang W, Zhou H, Ma BQ, Owiti A, Korban SS, Han YP. Divergent evolutionary pattern of sugar transporter genes is associated with the difference in sugar accumulation between grasses and eudicots. Sci Rep-Uk. 2016;6:29153.

37. Walker JC, Howard EA, Dennis ES, Peacock WJ. DNA-sequences required for anaerobic expression of the maize alcohol Dehydrogenase-1 gene. P Natl Acad Sci USA. 1987;84(19):6624-8.

38. Rosenkranz H, Vogel R, Greiner S, Rausch T. Wounded sugar beet (Beta Vulgaris L.) tap-root, hexose accumulation correlates with the induction of a vacuolar invertase isoform. J Exp Bot. 2001:52(365):2381-5.

39. Yamada K, Osakabe Y, Mizoi J, Nakashima K, Fujita Y, Shinozaki K, et al. Functional analysis of an arabidopsis thaliana abiotic stress-inducible facilitated diffusion transporter for monosaccharides. J Biol Chem. 2010;285(2):1138-46. 
40. Mizuno H, Kawahigashi $H$, Kawahara $Y$, Kanamori H, Ogata J, Minami H, et al. Global transcriptome analysis reveals distinct expression among duplicated genes during sorghum-interaction. BMC Plant Biol. 2012;12:121.

41. Mizuno H, Yazawa T, Kasuga S, Sawada Y, Ogata J, Ando T, et al. Expression level of a flavonoid 3'-hydroxylase gene determines pathogen-induced color variation in sorghum. BMC Res Notes. 2014;7:761.

42. Kong Y. Btrim: a fast, lightweight adapter and quality trimming program for next-generation sequencing technologies. Genomics. 2011;98(2):152-3.

43. Paterson AH, Bowers JE, Bruggmann R, Dubchak I, Grimwood J, Gundlach H, et al. The Sorghum Bicolor genome and the diversification of grasses. Nature. 2009;457(7229):551-6.

44. Kim D, Pertea G, Trapnell C, Pimentel H, Kelley R, Salzberg SL. TopHat2: accurate alignment of transcriptomes in the presence of insertions, deletions and gene fusions. Genome Biol. 2013;14:R36.

45. Trapnell C, Williams BA, Pertea G, Mortazavi A, Kwan G, van Baren MJ, et al. Transcript assembly and quantification by RNA-Seq reveals unannotated transcripts and isoform switching during cell differentiation. Nat Biotechnol. 2010;28(5):511-U174

46. Goodstein DM, Shu SQ, Howson R, Neupane R, Hayes RD, Fazo J, et al. Phytozome: a comparative platform for green plant genomics. Nucleic Acids Res. 2012;40(D1):D1178-D86.

47. Thimm O, Blasing O, Gibon Y, Nagel A, Meyer S, Kruger $P$, et al. MAPMAN: a user-driven tool to display genomics data sets onto diagrams of metabolic pathways and other biological processes. Plant J. 2004;37(6):914-39.

48. Jaiswal P, Ni J, Yap I, Ware D, Spooner W, Youens-Clark K, et al. Gramene: a bird's eye view of cereal genomes. Nucleic Acids Res. 2006;34(Database issue):D717-23.

\section{Submit your next manuscript to BioMed Central and we will help you at every step:}

- We accept pre-submission inquiries

- Our selector tool helps you to find the most relevant journal

- We provide round the clock customer support

- Convenient online submission

- Thorough peer review

- Inclusion in PubMed and all major indexing services

- Maximum visibility for your research

Submit your manuscript at www.biomedcentral.com/submit 